\title{
Effectiveness of Rocking Wall Tuned Mass Dampers in Reducing Dynamic Response of Structure Under Seismic Excitation
}

\section{Wei Nie}

Liaoning Technical University

Shuxian Liu

Liaoning Technical University

Shasha Lu ( $\sim$ lilyherb@163.com)

Liaoning Technical University

Shaodong Liu

Liaoning Technical University

Hang Yin

Liaoning Technical University

Chun Bai

Xuchang University

\section{Research Article}

Keywords: Rocking wall tuned mass dampers, Vibration control, Finite element analysis, Damping effect, Energy dissipation, effectiveness

Posted Date: October 25th, 2021

DOI: https://doi.org/10.21203/rs.3.rs-994696/v1

License: (c) (1) This work is licensed under a Creative Commons Attribution 4.0 International License.

Read Full License 


\title{
Effectiveness of rocking wall tuned mass dampers in reducing dynamic response of structure under seismic excitation
}

\author{
Wei Nie ${ }^{1 *}$, Shuxian Liu ${ }^{1}$, Shasha $\mathrm{Lu}^{1 *}$, Shaodong Liu ${ }^{1}$, Hang Yin ${ }^{1}$ and Chun Bai ${ }^{2}$ \\ 1 Department of Civil Engineering, Liaoning Technical University, Fuxin 123000, China \\ 2 College of Architecture and Civil Engineering, Xuchang University, Xuchang Henan 461000, China
}

\begin{abstract}
To improve the frequency sensitivity of a single tuned mass damper (STMD) and solve the accuracy problem of multiple tuned mass dampers (MTMD) in determining suitable installation location and effective number of tuned mass dampers (TMDs), this paper combines the construction characteristics of the rocking structures and the damping principle of TMDs to form a new type of MTMD, i.e., rocking wall tuned mass dampers (RW-TMDs). To verify the effectiveness of RW-TMDs, Finite element analyses were conducted to systematically compare the damping performance of the structure with STMD and RW-TMDs under different earthquake excitations. The results show that the RW-TMDs with different dynamic characteristics not only significantly suppress structural dynamic response but also improve the frequency sensitivity of TMD, reducing structural damage. Besides, the RW-TMDs can also improves structural interstory deformation pattern under white noise excitation, preventing the occurrence of the layer collapse mechanism. Overall, the RW-TMDs exhibit the damping effect of the MTMD system and the advantage of rocking structures to control inter-story deformation. Therefore, the RW-TMDs possess a high potential for practical applications for new and existing buildings.
\end{abstract}

Keywords Rocking wall tuned mass dampers. Vibration control. Finite element analysis. Damping effect. Energy dissipation- effectiveness

\section{Introduction}

Tuned Mass Damper (TMD), as a typical passive control technology, mainly consists of mass, springs, viscous dampers, or damping materials incorporated with a structural system. When the frequency of the TMD is same as or close to the natural frequency of the primary structure, the vibration energy of the main structure is transferred from the structure to the TMD due to resonance, and then the vibration energy is dissipated by the damping element ${ }^{1}$; so that the vibration response of the main structure is attenuated and the TMD is also called a "power absorber".

Frahm $^{2}$ first applied this design concept and achieved significant shock absorption, and then Yao ${ }^{3}$ introduced the concept of vibration control to civil engineering in 1972. Since then, various control strategies have been proposed by scholars, and their effectiveness in structural vibration control has been demonstrated. However, for a single tuned mass damper (STMD), the researches show that the TMD is most effective only when its frequency is close to or consistent with the fundamental frequency of the main structure ${ }^{4}$. When the parameters of the primary systems change slightly, the performance of TMDs will be greatly affected. It is a so-called off-tuning problem ${ }^{5}$, which has been investigated by researchers, such as, Setareh et al. ${ }^{6}$ and Kerschen et al. ${ }^{7}$. In addition, the large mass installed on the top of a tall building could result in concerns related to construction and building use ${ }^{8}$. Therefore, multiple tuned mass dampers (MTMDs) were developed to improve the effectiveness of STMD. Igusa and $\mathrm{Xu}{ }^{9}$ first proposed multi-tuned mass dampers (MTMDs) with distributed natural frequencies, and gave a seismic design formulation for MTMDs, demonstrating that MTMDs are much more robust than STMD. The effectiveness of MTMDs in vibration control has been subsequently demonstrated in numerous studies, such as those by Yamaguchi and Harnpornchai ${ }^{10}$, Rana and Soong ${ }^{11}, \mathrm{Li}^{12}$, Guo and Chen ${ }^{13}$. However, as scholars continued to study MTMDs in-depth, they also found that the effectiveness of the MTMDs mainly depends on the installation position of TMDs and effective number of TMDs.

When utilizing passive control techniques for shock absorption design, the optimal position that corresponds to the maximum modal amplitude needs to be determined for both STMD and MTMDs to install TMDs efficiently. Especially for MTMDs, the effectiveness of multi-mode control will be suppressed if the TMDs deviate from a suitable location. Petit, Loccufier, and Aeyels ${ }^{14}$ obtained a reasonable placement for TMDs based on a maximum shift in the structural frequency from the resonance forcing frequency. The results show that the damping effect can be significantly improved by installing TMDs effectively. Elisa and Matsagar ${ }^{4}$ also concluded that the performance of MTMDs can be improved if they are optimally distributed along with the height of the structure. Furthermore, the effectiveness of the STMD in vibration control under wind and seismic excitation was studied by Elias, Matsagar and Datta ${ }^{15}$, and the results show that if the STMD is installed in accordance with the corresponding modal shape, the different modal responses can be effectively controlled. Moon $^{8}$ demonstrated that increasing the number of TMDs in the MTMD system can improve the effectiveness of the 
MTMDs system; But due to the complexity of the structure, the optimal number of TMDs in the MTMDs requires further study. Although the robustness of the system to detuning can be improved by a wider frequency bandwidth, the performance of the MTMDs system may be suppressed due to the reduction in the total number of effective TMD units tuned to the target frequency ${ }^{10}$. Therefore, the main problem is that the placement of TMDs corresponding to the maximum modal amplitudes cannot be determined quickly and precisely, due to the random nature of seismic excitation, the frequency sensitivity of each TMD in the MTMDs, and the complexity of the structural design. In addition, a suitable number of TMDs in the MTMDs is difficult to precisely determine, for the additional TMDs changes the mode shape. If these uncertainties are ignored in practical engineering, the TMD will perform poorly due to mistuning ${ }^{16}$. In addition, the number of TMDs required for the practical application in MTMDs is generally higher than the theoretical number ${ }^{4}$. Furthermore, Moon also showed that the effectiveness loss of MTMDs is minimal if they are distributed vertically based on the mode shape ${ }^{8}$. In summary, for MTMD system, it is clear that the key issues are determining the effective number of TMDs and installing each TMD in a reasonable position to optimize damping effect. Therefore, it is necessary to design a new type of MTMDs that can both effectively improve STMD frequency sensitivity and conveniently exert the effect of multi-modal control.

Rocking structures are a popular type of vibration damping technology with various forms, such as free rocking structures, controlled rocking structures, and selfresetting structures. However, rocking structures, a kind of controlled rocking structure, has been rapidly developed because of their unique structural advantages. For example, self-centering systems can maintain building plumb after swing, for the self-weight and residual drifts can be effectively eliminated with swing deformation, and inelastic deformations can be concentrated in replaceable ductile fuses ${ }^{17}$. Consequently, Zhou and $\mathrm{Lu}{ }^{18}$ reported that the future development trend of rocking structures will place more emphasis on the seismic conceptual design of the overall structure, which facilitates multi-modal control of sub-structured systems. Besides, compared with the frame, the rocking wall generally has greater bending stiffnes $\mathrm{s}$ due to relax the bottom constraint of the wall, reducing the internal force requirement of the wall under earthquake. Thus, the rocking wall can be used as an auxiliary part of the frame to control the structural deformation mode and prevent the concentrated damage of the frame. In addition, the effect of damping and self-resetting of rocking structures under earthquake excitation has been demonstrated by many scholars through theoretical and experiments studies, such as those by Roh and Reinhorn ${ }^{19}$, Vassiliou and Makris ${ }^{20}$, Wada and $\mathrm{Qu}^{21}$ and $\mathrm{Qu}$ et al. ${ }^{22}$. A representative example is a case where the 11-story G3 building of the University of Tokyo in Japan was reinforced with the rocking walls survived a 9.0 magnitude earthquake 22. However, tuned mass dampers in the form of rocking walls have not been reported in the literature. Therefore, to better realize multi-mode control of MTMDs, this paper integrates the construction characteristics of rocking wall structure and the vibration attenuation mechanism of the TMD system to propose another form of MTMDs system, i.e. rocking wall tuned mass dampers (RW-TMDs). A pair of rocking walls were arranged symmetrically along the structural height to achieve structural multi-modal control. Each side of the rocking wall is connected in parallel with the main structure by dampers and springs on each floor. In addition, the RW-TMDs proposed in this paper can improve the frequency sensitivity of STMD and reduce the difficulty in determining control model of MTMDs. Moreover, the rocking wall with a bottom hinge can release the bending moment at the base of the wall and reduce the load-bearing capacity requirements for the wall and foundation.

Another key issue is that the effectiveness of TMDs is mainly dependent on the tuning frequency and optimum damping. Joshi and Jangid ${ }^{23}$ concluded that the optimization design of MTMDs system is more effective than the STMD system. In addition, Yamaguchi and Harnpornchai ${ }^{10}$ pointed out that compared with the parameter optimization of STMD, the robustness and effectiveness of MTMDs cannot be achieved simultaneously, i.e., the most effective MTMD is not very robust. However, the design formula of the bituned mass dampers proposed by Ok, Song and Park ${ }^{24}$ can ensure good effectiveness and robustness under seismic excitations.

Therefore, the intention of the present study is to investigate the effectiveness of a TMD in the form of a pinned rocking wall. To better exert the damping effect of RW-TMDs, with reference to the literature ${ }^{24}$, the first mode was used as the control mode to design the optimized parameters, and the optimal frequency ratio is taken to be 1 . Moreover, the STMD with the same mass ratio (5\%) was designed by the Den-Hartog design formula to check the rationality of the system. Then, the vibration control effects of the six-story RC frame structures with STMD or RWTMDs under Gaussian white noise and three different seismic waves were compared and analyzed by numerical simulations. The results show that the RW-TMDs exhibit a better and more stable damping performance than that of the STMD overall. However, the RW-TMDs are a crossover study within two fields of recoverable functional structures and passive control. They have the damping effect of the MTMDs system and the advantage of rocking structures to control inter-story deformation. Therefore, the RW-TMDs possess a high potential for practical applications for new buildings and buildings in service.

\section{Theoretical analysis of the RW-TMDs}

\subsection{Mathematical models}


To simplify the calculations, the following assumptions are made. (1) The rocking wall is assumed to be uniform rigid body, and the displacement of the RW-TMDs along the height of the structure presents an inverted triangular distribution. (2) Structural systems are excited by unidirectional seismic excitation. (3) The effects of the soilstructure-interaction (SSI) are not taken into consideration. (4) The thickness and length of the RW-TMDs are much smaller than the height. The mechanical model is shown in Fig. 1.

The equations of motion that govern the response of the structure when subjected to ground excitation can be expressed as:

$$
\begin{gathered}
m_{1}+\left(c_{1}+c_{2}\right) \&+\left(k_{1}+k_{2}\right) x_{1}-c_{2}-k_{2} x_{2}- \\
c_{d 1}\left(h_{1}-k_{d 1}\left(h_{1} x_{d}-x_{1}\right)=-m_{1}(t)\right. \\
m_{2}-c_{2}+\left(c_{2}+c_{3}\right)-c_{3}-k_{2} x_{1}+\left(k_{2}+k_{3}\right) x_{2} \\
-k_{3} x_{3}-c_{d 2}\left(h_{2}-k_{d 2}\left(h_{2} x_{d}-x_{2}\right)=-m_{2}(t)\right.
\end{gathered}
$$$$
\mathrm{M}
$$

$$
\begin{gathered}
m_{6}+c_{6}(-)+k_{6}\left(x_{6}-x_{5}\right)- \\
c_{d 6}\left(h-k_{d 6}\left(h_{x_{d}}-x_{6}\right)=-m_{6}(t)\right. \\
I_{0}+c_{d i} h_{i}^{2} \&-c_{d i} h_{i} x_{i}+k_{d i} h_{i}^{2} x_{d}-k_{d i} h_{i} x_{i}=0
\end{gathered}
$$

where $i=1,2, \mathrm{~L}, 6 ; I_{0}=\frac{m_{d} h^{2}}{3} ;$ The $\mathrm{h}$ is the height of rocking wall that equal to the total height of building and $h_{i}$ is floor-to-floor height. The $I_{0}$ and $m_{d}$ are the moment of inertia and mass of RW-TMDs. The $k_{d i}$ and $c_{d i}$ are the stiffness and damping of the ith layer of RW-TMDs. The $m_{i}$, $k_{i}$ and $c_{i}$ are the mass, stiffness and damping of the ith layer of the RC frame structure. $x_{i}, \ldots$ are the displacement, velocity, and acceleration vectors of the structural relative to the ground; $x_{d}$ is the rotation angle of RW-TMDs.

$(t)$ is the acceleration of the input ground motion. In addition, the Rayleigh damping is considered as a linear combination of mass and stiffness ${ }^{25}$.

Writing the above equations in matrix form is expressed as:

$$
\begin{aligned}
& {[M]\{+[C]\{d,+[K]\{x\}=} \\
& {\left[C_{d}\right]\left\{h_{i}\right\}+\left[K_{d}\right]\left\{h_{i}\right\} x_{d}-[M]\{I\}(t)}
\end{aligned}
$$

$I_{0}+\left\{h_{i}\right\}\left[C_{d}\right]\left(\left\{h_{i}\right\} \mathbb{d}_{d}-\{d\}\right)+\left\{h_{i}\right\}\left[K_{d}\right]\left(\left\{h_{i}\right\} x_{d}-\{x\}\right)=0(6)$

where $[\mathrm{M}],[\mathrm{K}],[\mathrm{C}]$ are the mass matrix, stiffness matrix and damping matrix of structure, $\{I\}$ is the unit matrix; $\left[\mathrm{K}_{\mathrm{d}}\right]$ and $\left[\mathrm{C}_{\mathrm{d}}\right]$ are the stiffness matrix and damping matrix of the RW-TMDs and they are expressed as follow:
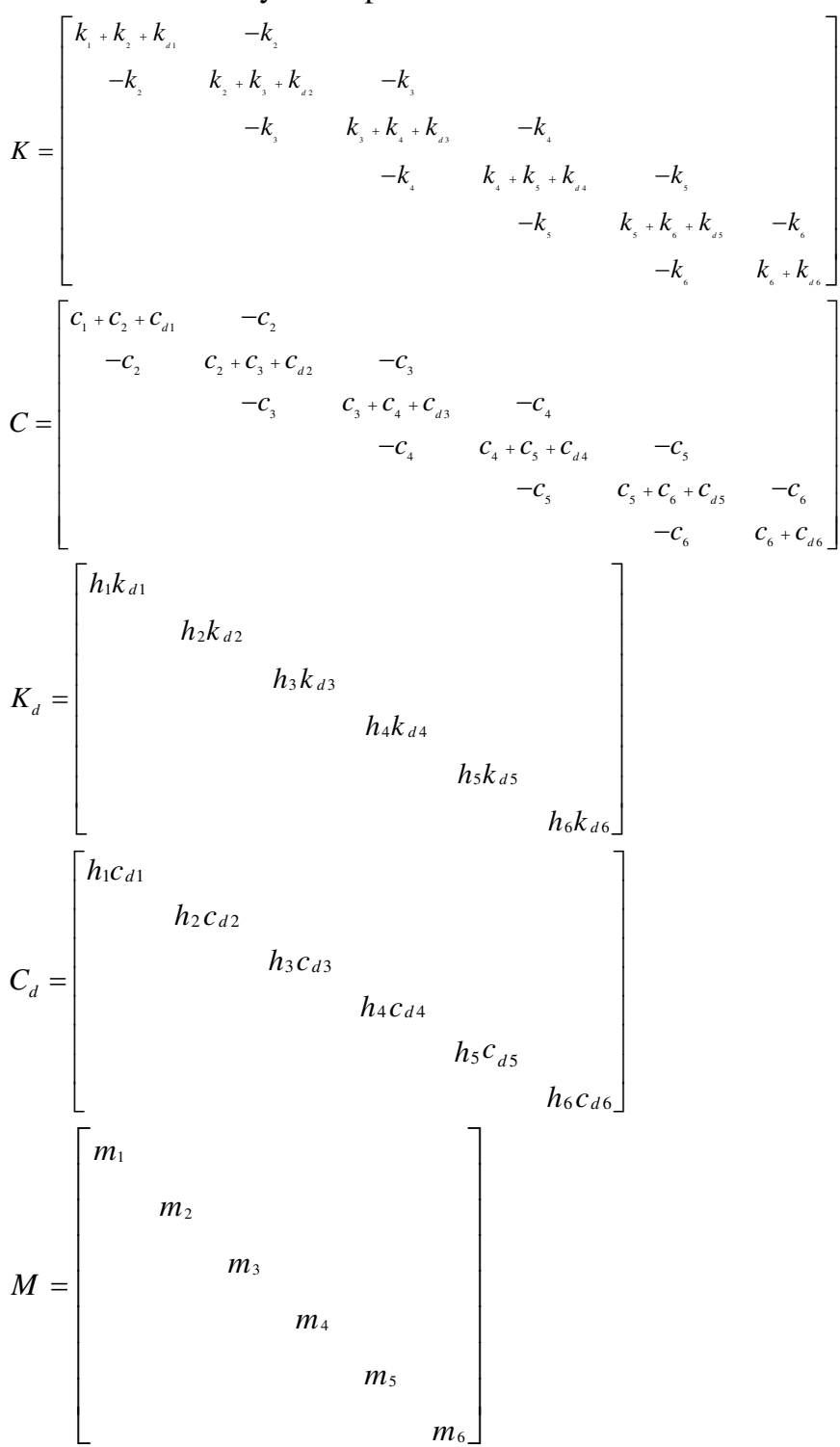

$\{x\}=\left\{x_{1}, x_{2}, x_{3}, x_{4}, x_{5}, x_{6}\right\}^{T}$

$\left\{h_{i}\right\}=\left\{h_{1}, h_{2}, h_{3}, h_{4}, h_{5}, h_{6}\right\}^{T}$.

Let $\{x(t)\}=[\Phi]\{q(t)\}=\sum_{i=1}^{6} q_{i}(t) \varphi_{i}$

where $[\Phi]$ is the vector matrix of structural eigenvector; and $\{q(t)\}=\left\{q_{1}(t), q_{2}(t), q_{3}(t), q_{4}(t), q_{5}(t), q_{6}(t)\right\}^{T}$ is the column vector of generalized coordinates.

Assuming that the stiffness and damping of the each RW-TMD are same to design and construct conveniently, that is $k_{d 1}=k_{d 2}=k_{d 3}=k_{d 4}=k_{d 5}=k_{d 6}$ ， 
$c_{d 1}=c_{d 2}=c_{d 3}=c_{d 4}=c_{d 5}=c_{d 6}$. The equivalent stiffness and damping of RW-TMD are express by $k_{d}$ and $c_{d}$. And $\omega_{d}^{2}=\frac{k_{d}}{m_{d}}, 2 \xi_{d} m_{d}=\frac{c_{d}}{m_{d}}$.

Substituting the Eq (7) into the Eqs (5)-(6) gets the following express:

$$
\begin{aligned}
& \{t)\}+\left[C^{*}\right]\{d(t)\}+\left[K^{*}\right]\{q\}= \\
& -\left\{\gamma_{d}\right\}\left(c_{d} \&+k_{d} x_{d}\right)-\{\gamma\}(t)
\end{aligned}
$$

$$
+\frac{6}{h^{2}} \xi_{d} \omega_{d} h_{i}\left(h_{i}-\frac{3}{h^{2}} \omega_{d}^{2} h_{i}\left(h_{i} x_{d}-x_{i}\right)=0\right.
$$

where $\{\gamma\}=\left[M^{*}\right]^{-1}[\Phi]^{T}[M]\{I\}$,

$\left\{\gamma_{d}\right\}=\left[M^{*}\right]^{-1}[\Phi]^{T}\left\{h_{j}\right\},\left[M^{*}\right]=[\Phi]^{T}[M][\Phi]$

$\left[C^{*}\right]=\left[M^{\prime}\right]^{-1}[\Phi]^{T}[C][\Phi] ;\left[K^{*}\right]=\left[M^{\prime}\right]^{-1}[\Phi]^{T}[K][\Phi]$.

In the generalized coordinates, the multi-degree-offreedom equations in $\mathrm{Eq}$ (8) are mutually independent, and the jth equation is as follow.

$\oiint_{j}+2 \xi_{j} \omega_{j} \&_{J}+\omega_{j}^{2} q_{j}=\mu_{j} h_{i} \Phi_{i j}\left[2 \xi_{d} \omega_{d} \&_{d}^{2} x_{d}\right]$

$-\gamma_{j}(t)(j=1,2, \mathrm{~L}, 6)$

where $\mu_{j}=\frac{m_{d}}{m_{j}{ }^{*}}, \gamma_{j}=\frac{\sum_{j=1}^{6} m_{j} \Phi_{i j}}{m_{j}^{*}}$ are the mass ratio and mode-participation coefficient of $j$ th mode.

$m_{j}^{*}=\sum_{j=1}^{6} m_{j} \Phi_{i j}^{2}$ is the generalized mass of jth mode.

$\Phi_{i j}$ is the mode vector of ith element in jth mode.

When structural seismic response is controlled by a certain mode (jth mode), only the influence of $j$ th mode on the TMD system is considered, and the Eq (9) can be written as follow:

$$
\begin{aligned}
& +\frac{6}{h^{2}} \xi_{d} \omega_{d} h_{i}^{2} \&+\frac{3}{h^{2}} \omega_{d}^{2} h_{i}^{2} x_{d}= \\
& \frac{6}{h^{2}} \xi_{d} \omega_{d} h_{i} \Phi_{i j} \&+\frac{3}{h^{2}} \omega_{d}^{2} h_{i} \Phi_{i j} q
\end{aligned}
$$

Let $(t)=-e^{i \omega t}, q_{j}(t)=H_{j}(\omega) e^{i \omega t}, x_{d}(\mathrm{t})=H_{d}(\omega) e^{i \omega t}$, $f_{j}=\frac{\omega_{d}}{\omega_{j}}, \lambda=\frac{\omega}{\omega_{j}}, \alpha=\frac{h_{i}}{h}$.

The transfer function expression of the structure can be obtained by substituting them into Eq (10)-(11).

$$
\begin{gathered}
H_{j}(\omega)=\frac{1}{d}\left[\left(3 f_{j} \alpha^{2} h^{2}\left(2 i \lambda \xi_{d}+f_{j}\right)-h^{2} \lambda^{2}\right]\right. \\
H_{d}(\omega)=\frac{1}{d}\left[\frac{3}{h} \gamma_{j} \Phi_{i j} \alpha f_{j}\left(2 i \lambda \xi_{d}+f_{j}\right)\right]
\end{gathered}
$$

where

$$
d=\omega_{j}^{2}\left\{\begin{array}{l}
{\left[3 f_{j} \alpha^{2} h^{2}\left(2 i \lambda \xi_{d}+f_{j}\right)-h^{2} \lambda^{2}\right]\left(1-\lambda^{2}+2 i \lambda \xi_{j}\right)} \\
-3\left[\mu_{j} \alpha \Phi_{i j} f_{j}\left(2 i \lambda \xi_{d}+f_{j}\right)\right]^{2}
\end{array}\right\}
$$

and the $\omega$ represents frequency of external excitation.

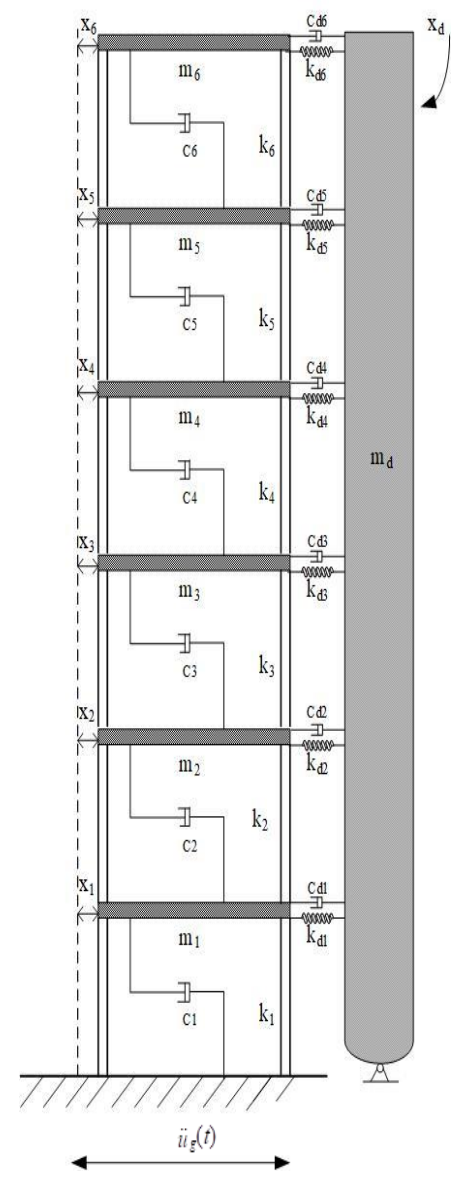

Fig. 1 Calculation model of the RW-TMDs.

Since the value of the frequency, damping ratio, mass, and mode shape have an effect on the transfer function, as shown in Eqs (12)-(13), Yamaguchi and Harnpornchai 10 pointed out that to improve the energy dissipation capacity and the vibration control effect of TMDs, it is necessary to adjust the frequency of TMDs to match the structural natural frequency and select the appropriate number of TMDs. However, Domizio, Ambrosini and Curadellii ${ }^{26}$ found that adding TMDs would be ineffective if the frequency content of the earthquake differs from the frequency of the fundamental mode. Therefore, to verify the 
effectiveness of RW-TMDs, the frequency ratio $\left(f_{j}=\frac{\omega_{d}}{\omega_{j}}\right)$ is taken as 1 in this paper, and the specific parameter design is shown in the following section.

\subsection{Designing the RW-TMDs}

The theoretical analysis of the RW-TMDs shows that the parameter design is very important to its effectiveness. In this paper, the structural maximum displacement is selected as the design target, and two RW-TMDs with different dynamic characteristics are arranged along with the building height. For convenience, the following assumptions are made: (1) The first modal frequency of the structure is selected as the controlled frequency; (2) The bandwidth does not cross other structural frequencies; (3) The RW-TMDs has the same mass. The RC frame structure model (as show in Fig. 2) is a six-story frame and the RWTMDs are symmetrically distributed along the x-direction of the structure. Therefore, referring to the parameter design of the MTMDs in the literature ${ }^{12,27}$, the average frequency of two RW-TMDs is $\omega_{D}=\frac{\omega_{d-1}+\omega_{d-2}}{2}$ (14), where $\omega_{d-1}$ and $\omega_{d-2}$ are the frequency of the first and second RW-TMD. The parameter $\beta$ is the nondimensional frequency bandwidth of the RW-TMDs defined as $\beta=\frac{\omega_{d-2}-\omega_{d-1}}{\omega_{D}}$ (15). Thus, the natural frequency of the each TMD is expressed as

$\omega_{d-g}=\omega_{D}\left[1+\left(g-\frac{n+1}{2}\right) \frac{\beta}{n-1}\right] n=2, \quad g=1,2$

The frequency ratio of each RW-TMDs is

$$
f_{d-g}=\frac{\omega_{d-g}}{\omega_{s}}=f\left[1+\left(g-\frac{n+1}{2}\right) \frac{\beta}{n-1}\right]
$$

where $\omega_{d-\mathrm{g}}$ denotes the frequency of the gth RWTMDs, and $\omega_{s}$ is the first mode frequency. The damping ratio of the gth RW-TMDs is $\xi_{d-g}=f_{d-g} \xi_{D}$ (18), where $\xi_{D}=\left(\sum_{g=1}^{n} \xi_{d-g}\right) / n$ is the average damping ratio of two RW-TMDs. Therefore, the stiffness and damping coefficients of the gth RW-TMDs can be calculated.

In addition, Hwang, Lee and Kareem ${ }^{28}$ showed that only the first mode mass of the structure is used for the design of TMDs, regardless of the mass of the other modes if the frequency of the TMD is tuned to the first modal frequency of the primary structure. Besides, Tharwatand Sakr ${ }^{29}$ and Matta ${ }^{30}$ had shown that the effectiveness of MTMDs improves with increasing mass ratio. Therefore, the total mass of RW-TMDs has been considered to be $5 \%$ of the first modal mass of structure $\left(m_{s}=294.63 \times 10^{3} \mathrm{~kg}\right)$. In addition, to verify the effectiveness of the RW-TMDs, a STMD with the same mass ratio was designed for comparative analysis. The optimal frequency ratio is taken to be 1, i.e., $f=\omega_{d-g} / \omega_{s}=1$. However, many studies have shown that the most effective MTMD is not very robust. Therefore, to verify the effectiveness of RW-TMDs, the optimized parameters given in reference ${ }^{24}$ with better robustness and effectiveness are selected. The damping ratio of the first RW-TMD is $\xi_{d-1}^{r w}=0.2623 \mu^{0.3386}=0.0951$; the damping ratio of the second RW-TMD is $\xi_{d-2}^{r w}=0.4054 \mu^{0.46}=0.1022$; and the damping ratio of the STMD is $\xi_{\text {opt }}^{\text {tmd }}=\sqrt{\frac{3 \mu}{8(1+\mu)}}=0.1336$.

Therefore, Table 1 shows the design parameters of the TMDs system.

Table 1 Properties of tuned mass dampers.

\begin{tabular}{ccccc}
\hline \multicolumn{1}{c}{ Mass(kg) } & $\begin{array}{c}\text { Stiffness } \\
(\mathrm{kN} / \mathrm{m})\end{array}$ & $\begin{array}{c}\text { Damping } \\
(\mathrm{kN} . \mathrm{s} / \mathrm{m})\end{array}$ \\
\hline \multirow{2}{*}{ RW-TMDs } & TMD $_{\mathrm{d}-1}$ & 7365.75 & 3448.029 & 1.516 \\
& & & & \\
& TMD $_{\mathrm{d}-2}$ & 7365.75 & 3448.029 & 1.629 \\
STMD & TMD & 14731.50 & 6896.058 & 4.259
\end{tabular}

Each RW-TMD is connected in parallel to the main structure by six pairs identical springs and dampers. Therefore, compared to the STMD, the RW-TMDs can disperse the total stiffness and damping to each small member, which can prevent the failure of a certain component from overall mistuning of the TMDs system. Therefore, the springs and dampers of the first and second RW-TMD are $287.3358 \mathrm{kN} / \mathrm{m} \& 0.1263 \mathrm{kN} . \mathrm{s} / \mathrm{m}$ and $287.3358 \mathrm{kN} / \mathrm{m} \& 0.1358 \mathrm{kN} . \mathrm{s} / \mathrm{m}$, respectively.

\subsection{Dimension design of the RW-TMDs}

It was found that the stiffness of the rocking wall plays a controlling role on the structural deformation mode.

Based on the results of steel structures studied by MacRae et al. ${ }^{31}, \mathrm{Qu}^{32}$ gave an expression for the stiffness coefficient $(\alpha)$ to measure the relative stiffness of the rocking wall in the rocking wall- frame structure.

$$
\alpha=\frac{E I}{k h_{f}^{3}}
$$

where, $E I$ is the flexural stiffness of the rocking wall section; $k$ is the layer shear stiffness of the frame 

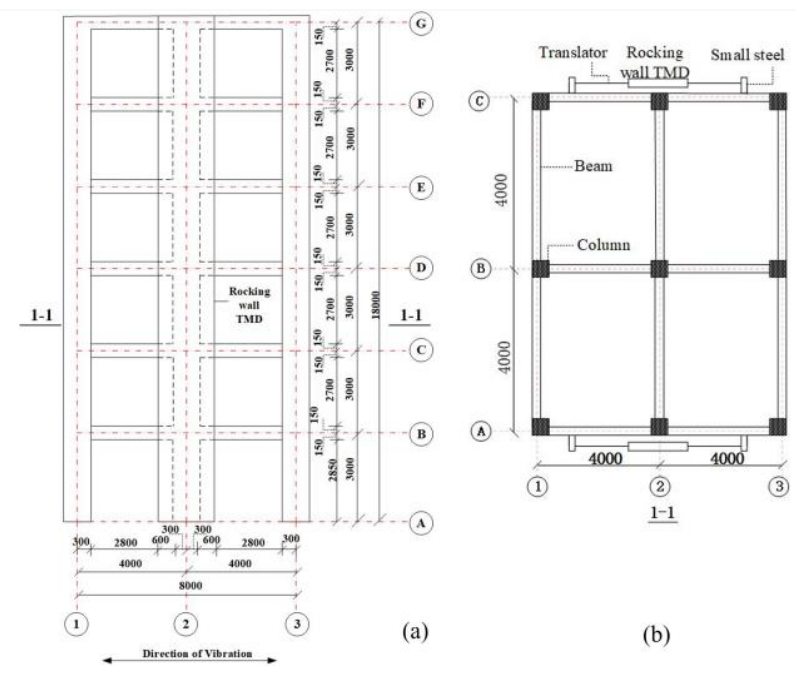

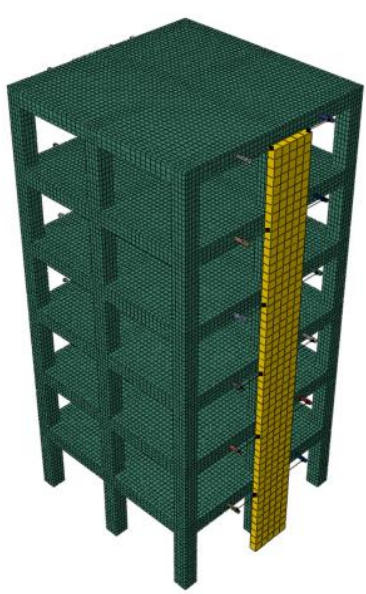

(c)

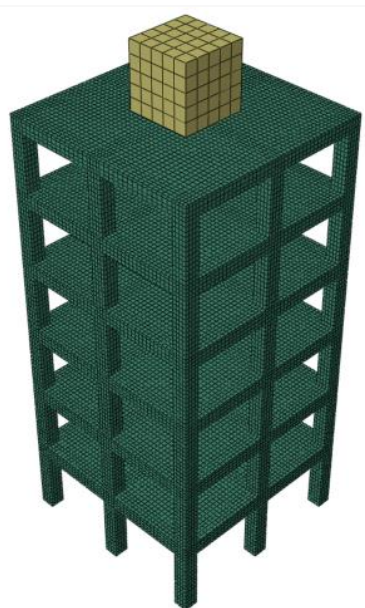

(d)

Fig. 2 Configuration of the frame model with RW-TMDs (unit: mm). (a) Elevation; (b) floor plan; and (c)-(d) finite element analytical model of RW-TMDs and STMD.

structure; and $h_{f}$ is the story height. However, there are significant difference in the characteristics of steel and RC frame structures. Therefore, Yang et al. ${ }^{33}$ defined the stiffness ratio (as expressed in Eq. (20) of rocking wall to frame structure with reference to the stiffness ratio of frame and shear wall in shear wall-frame system and the definition of stiffness eigenvalues.

$$
\delta=\frac{E_{\omega} I_{\omega}}{G_{f} h^{2}}
$$

where, $E_{\omega} I_{\omega}$ is the flexural stiffness of the rocking wall section; $\delta$ is the stiffness ratio of rocking wall to frame; $h$ is the total height of the structure;

$$
G_{f}=\frac{\sum_{i=1}^{n} D_{i} h_{i}^{2}}{\sum_{i=1}^{n} h_{i}}
$$

where, $D_{i}$ is the shear stiffness of the ith layer calculated using the D-value method. $h_{i}$ is the height of the ith layer; $\mathrm{n}$ is the number of structural layers.

Yang et al. ${ }^{33}$ reported that the structure has various failure mode and failure mechanisms at different stiffness ratios $(1.27 \%, 2.48 \%$ and $6.81 \%)$. Chen and Huang 34 suggested there are an upper bound of mass ratio (15\%) for the TMDs. Because the rocking wall under the stiffness ratio of $2.48 \%$ begins to change the failure mechanism of the frame structure, gradually changing from the layer failure mechanism to the overall failure mechanism. Therefore, the stiffness ratio of $2.48 \%$ is used as an index to design the size of rocking wall that is $300 \mathrm{~mm} \times 1800 \mathrm{~mm} \times 18000 \mathrm{~mm}$. To verify the effectiveness of the RW-TMDs, a finite element analysis (FEA) was carried out in the next chapter.

\section{Finite element analysis of the RW-TMDs}

According to the parameter design in Section 2.2 and 2.3, six-story RC frame structures with and without TMDs were established in the present study by the finite element analysis software ABAQUS.

The structural model is a two-span, six-story symmetrical frame structure that is regular in plane and elevation. The floor dimension is $8000 \mathrm{~mm} \times 8000 \mathrm{~mm}$, the floor-to-floor height is $3000 \mathrm{~mm}$, and the thickness of the floor slab is $120 \mathrm{~mm}$. The cross-sectional size of the column and beam are $600 \mathrm{~mm} \times 600 \mathrm{~mm}$ and $300 \mathrm{~mm} \times 600 \mathrm{~mm}$, respectively. While the size of the rocking walls is $300 \mathrm{~mm} \times 1800 \mathrm{~mm} \times 18000 \mathrm{~mm}$, as shown in Fig. 2(a-b). The types of rebar and stirrup are HRB335 and HPB300, respectively. The density and modulus of elasticity $(\mathrm{Ec})$ of concrete are assumed to be $2500 \mathrm{~kg} / \mathrm{m}^{3}$ and $2.98 \times 10^{10} \mathrm{~N} / \mathrm{m}^{2}$, respectively. The RW-TMDs set along with the height of the structure as shown in Fig. 2(c), while STMD is put on the roof of the structure as shown in Fig. 2(d). The plastic damage model was adopted for concrete, and ideal elastoplastic constitutive relationship was used for rebar. The concrete and steel reinforcement were simulated with solid elements (C3D8R) and truss elements (T3D2), respectively. The interaction between the steel reinforcement and the concrete was embedded, regardless of the slip between them. To verify the effectiveness of the RW-TMDs, a STMD was designed according to the same mass ratio, and the RW-TMDs and the STMD were simulated with solid elements (C3D8R).

\subsection{Difficulties in designing the RW-TMDs}

The good damping effect of rocking wall-frame structure has been proved by many scholars. In the actual engineering design, the bottom of rocking wall connects with the ground beam through certain connector, and then the rocking wall 
connects with the frame columns via metal yield-type dampers. The reason is that the rocking wall can increase relative displacement of certain parts when swaying occurs under external excitation, which provides the possibility to install energy-consuming components and increase structural energy consumption. However, many studies have shown that there are two difficulties in the design of rocking wall structures. one is the hinged connection at the bottom of the rocking wall, and the other is the connection member between the rocking wall and the frame structure. There have been many ways to design the hinged connection of the rocking wall and $\mathrm{Nie}$ et al $^{35}$ also designed a hinged support in the shaking table test to convenient disassembly. Besides, the damping principle of the TMD needs to be considered in the design of the RW-TMDs, for the vibration of the TMD system is driven by the main structure under external excitation. Therefore, a hinged base was made for the rocking wall and then connected to the bottom steel beam of the frame structure in literature ${ }^{35}$. In this way, the vibration of the main structure can drive the vibration of the auxiliary structure, so that the effect of out-of-phase vibration effectively improved. Correspondingly, in the FEA, the acceleration peaks of the three seismic waves (EL Centro wave, Taft wave and Artificial wave) were directly applied to the sections of all columns at the bottom of the frame structure except the rocking wall, but the bottom of the RW-TMDs need to be set as hinged connection.

Another design difficulty in the RW-TMDs is the connection between the rocking wall and the frame structure. Therefore, in the FEA, the main structure and the substructure are connected via a translator that regards as the spring-damper element in finite element software ABAQUS. Moreover, only linear stiffness and damping are considered in the parameter design of translator. And since the seismic excitation is unidirectional, the corresponding stiffness and damping coefficients of translator (reference to section 2.1) were only set in the direction of the seismic excitation. The translator connected with the frame through small steel $(100 \mathrm{~mm} \times 100 \mathrm{~mm} \times 600 \mathrm{~mm})$ that connected with frame beam via tie constraint to simulate the effect of embedded parts in the actual engineering field. Then, the reference point 1 (as shown in Fig. 3) that coupled with the small steel connected with reference point 2 that located at the height of each layer in rocking wall through a translator. Besides, it is necessary to ensure the reference point 1 and the reference point 2 at the same height to avoid the damage to the connectors caused by misaligned connection.

For convenience, the uncontrolled structures mentioned below are abbreviated as F model, and the structures with the STMD and RW-TMDs are abbreviated as FT model and FR model, respectively.

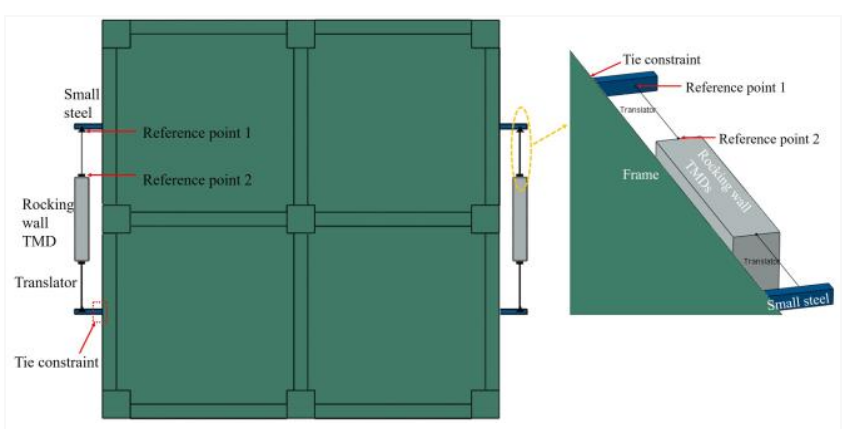

Fig. 3 Finite element model of RW-TMDs connector.

\subsection{Modal analysis}

In this paper, the modal analysis of the six-layer $\mathrm{RC}$ frame structure was performed through the subspace iterative method. Besides, Au-Yeung et $a l^{36}$ reported that the distribution of vibration energy is mostly within structural first few low frequency resonant modes. To demonstrate the multi-modal control effect of RW-TMDs, the first three modes are selected for analysis, as shown in Table 2.

Table 2 Comparative analysis of the first three modal frequencies of the structures

\begin{tabular}{cccccc}
\hline Mode No F model & $\begin{array}{c}\text { FR } \\
\text { model }\end{array}$ & $\begin{array}{c}\text { FT } \\
\text { model }\end{array}$ & $R_{f}(F R) \%$ & $R_{f}(F T) \%$ \\
\hline First & 3.4435 & 3.5747 & 2.9143 & -3.81 & 15.37 \\
Second & 3.4436 & 4.3441 & 3.2762 & -26.15 & 4.86 \\
Third & 4.2333 & 4.7689 & 4.0530 & -12.65 & 4.26
\end{tabular}

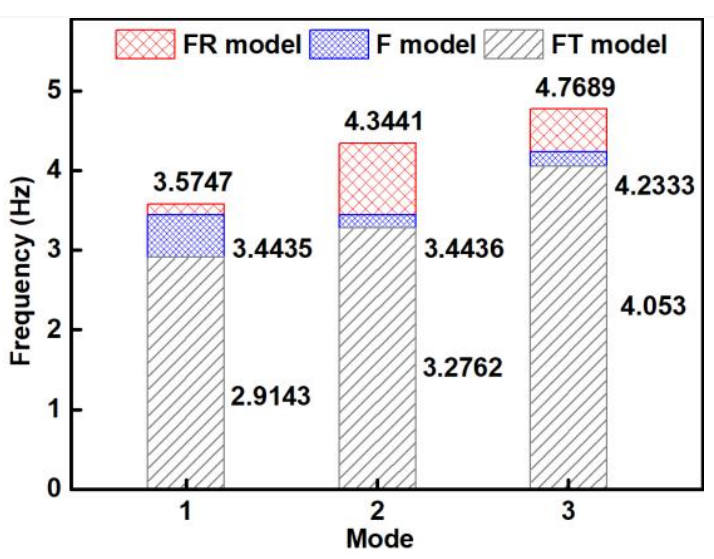

Fig. 4 The first three frequencies of controlled and uncontrolled structures. 


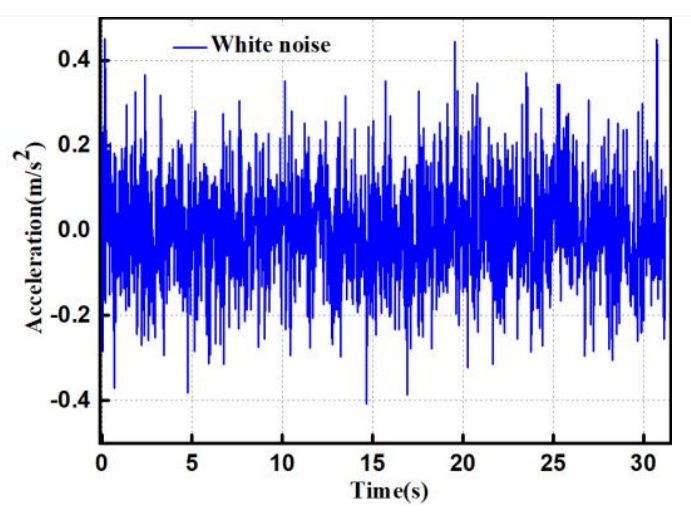

(a)Time history excitation

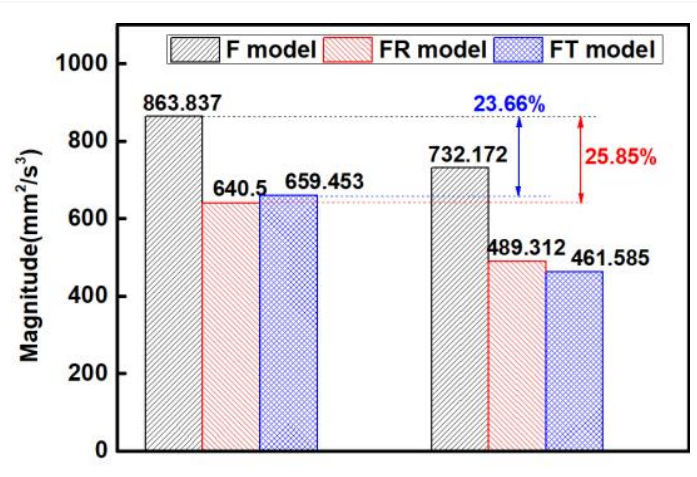

(c) Maximum peak of the frequency response curve



(b) Frequency response curve

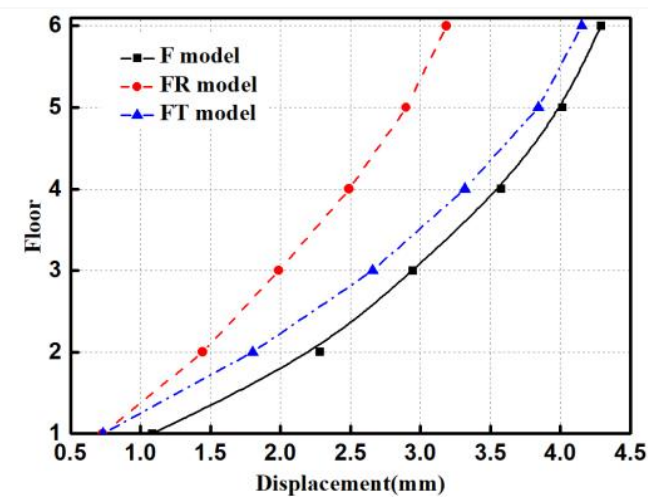

(d) Maximum lateral displacement

Fig. 5 Response of the controlled structures and uncontrolled structure under white noise

In addition, the shift in the frequency $\left(R_{f}\right)$ is defined to demonstrate the influence of the damping system on the structural vibration frequency, that is

$$
R_{f}=\left(1-\frac{f_{i}}{f_{s i}}\right) \times 100 \% \quad i=1 \text { to } n
$$

where $f_{s i}$ and $f_{i}$ are the vibration frequencies of the uncontrolled and controlled structures, respectively.

Table 2 shows that the $\left|R_{f}\right|$ value of the first modal frequency of the STMD system $(15.37 \%)$ is larger than that of the RW-TMDs system $(3.81 \%)$, which is consistent with the expected results. To intuitively demonstrate the frequency offset of the first three modes, the frequency comparison between the controlled and uncontrolled structures is shown in Fig. 4. In addition, Table 2 shows that the $R_{f}$ values of the second and third modes of the FR model and FT model are $-26.15 \%$ \& $4.86 \%$ and $-12.65 \%$ \& $4.26 \%$ respectively. Consequently, the control effect of STMD is significant for the first mode, while the RWTMDs system has the advantage of multi-mode control, especially for the second mode, which is consistent with the phenomenon observed in literature ${ }^{37,38}$. Therefore, to further investigate the effectiveness of RW-TMDs, a comparative analysis of the damping effect of the controlled structure under seismic wave excitations will be performed.

\subsection{Vibration-reduction performance under Gauss white noise excitation}

To verify the effectiveness of the RW-TMDs, the controlled and uncontrolled structures were subjected to broadband white noise wave. White noise is one of the general types of vibration testing services performed for the structure. Fig. 5 (a-d) shows the time history of white noise excitation with PGA $=0.05 \mathrm{~g}$, the frequency response curve $(\mathrm{FRC})$ at the top floor, the first two peaks on the FRC, and the maximal lateral displacement of the controlled and uncontrolled structures. The effectiveness index of RW-TMDs is defined as the peak of the FRC, while the smaller peak value characterizes better vibration control.

Fig. 5 (b-c) shows that the peak value of FRC decreases significantly compared with uncontrolled structure, indicating the effectiveness of the controlled structure. Furthermore, Fig. 5 (b) shows that the maximum peak of the controlled structure appears near the fundamental frequency of the structure, while the frequency corresponding to the first peak of the FR model becomes larger, consistent with the results of modal analysis. This is mainly because the RW-TMDs laying along the height of the structure have the effect of multi-modal control and multiple TMDs change the dynamic characteristics of the main structure, widening the tuning frequency range of the 


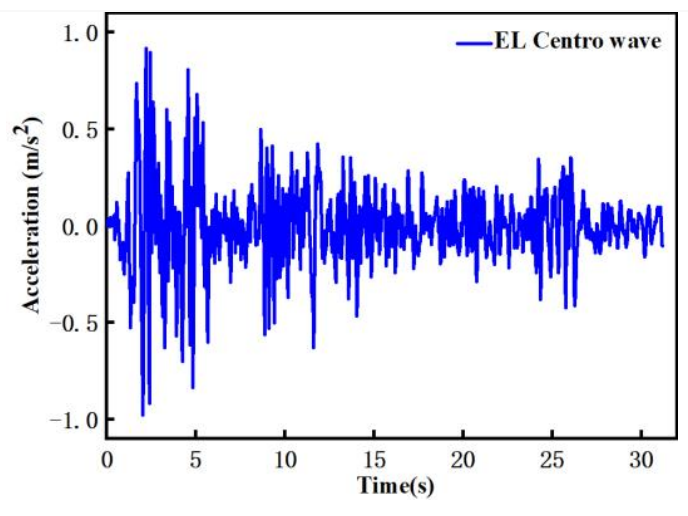

(a) EL Centro wave

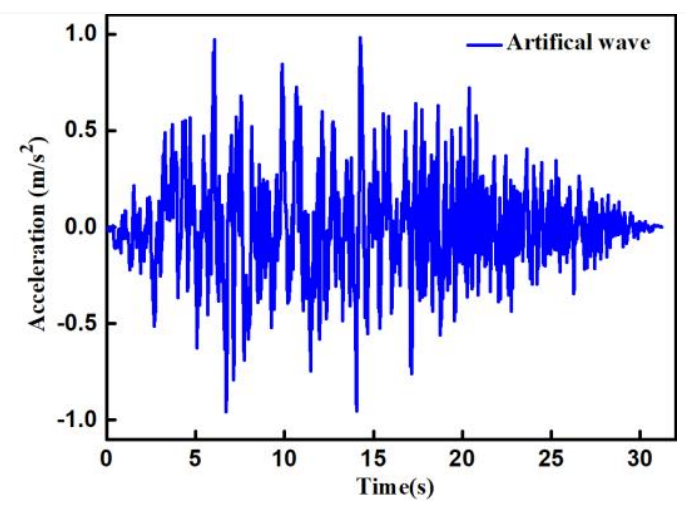

(c) Artifical wave

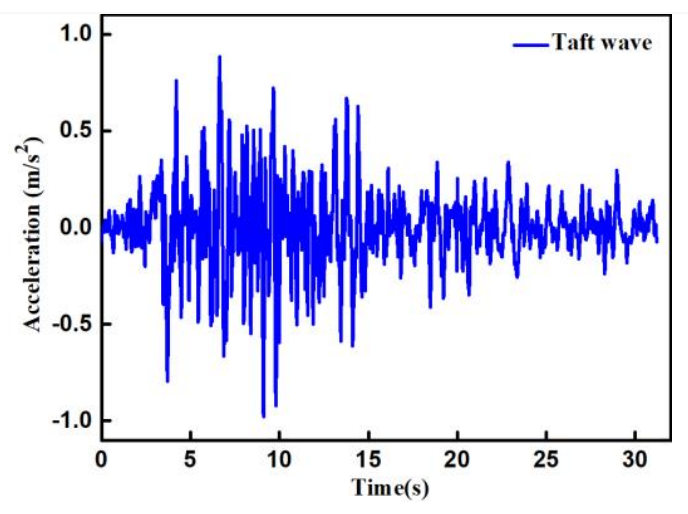

(b) Taft wave

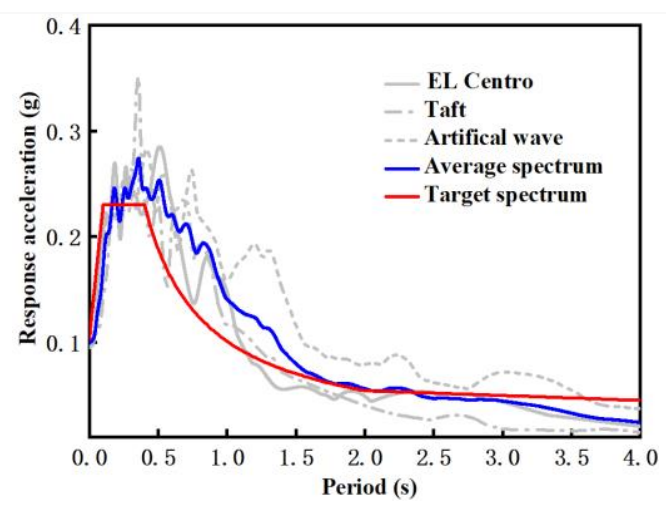

(d) Response spectrum of the input ground motions

Fig. 6 Accelerations and response spectrum of input waves

TMDs system. Compared with the F model, the first-peak reduction rates of the FR model and FT model are $25.85 \%$ and $23.66 \%$, respectively, as shown in Fig. 5(c). While the second-peak reduction rates of the FR model and FT model are $33.17 \%$ and $36.96 \%$, respectively, indicating that RWTMDs are more effective and have comparable vibration control effects with STMD under broadband white noise excitation. In addition, Fig. 5(d) shows that the damping effect improved with the height increased under white noise, and the maximum lateral displacement of the FR model is significantly lower than that of the FT model, demonstrating the effectiveness of the multi-modal control of the RW-TMDs. Moreover, the trend of inter-story displacement of the FR model is more uniform than that of the F model and FT model. Conversely, the F model and FT model present typical shear deformation characteristics of frame structure whose deformation exhibit a concave shape. Therefore, this reflects the advantages of the rocking wall structure system that improves the inter-story deformation pattern, making the inter-story displacement tend to be uniform and then preventing the occurrence of the layer collapse mechanism. These advantages have been proven by many researchers such as Qu et al. ${ }^{22}$.

Overall, it can be concluded that the RW-TMDs have a significant contribution in decreasing the vibration response of the structure under white noise. A great number of studies have shown that the effectiveness of the TMDs system is greatly affected by the characteristics of seismic waves, but white noise excitation is not the actual seismic excitation. Therefore, to better verify the effectiveness of the RW-TMDs, three different characteristics of seismic excitation are selected for the controlled and uncontrolled structures in the later part of this study.

\subsection{Vibration-reduction performance under different seismic waves}

To further prove the seismic performance of RW-TMDs under realistic earthquakes excitations, the structure was subjected to EL Centro, Taft, and Artificial ground accelerations (as shown in Fig. 6(a)-(c)), which were adjusted according to precautionary earthquakes with seismic intensities of 7 degrees $(0.1 \mathrm{~g})$ and 8 degrees $(0.2 \mathrm{~g})$ of the Chinese design $\operatorname{code}^{39}$, while the spectrum components of three seismic waves were sufficient for this study ${ }^{40}$, as shown in Fig. 6(d). A maximum deviation of the average spectrum of three records from the target spectrum was imposed within $10 \%$ in the period range of interest ${ }^{41}$. 
Table 3 Acceleration responses at the roof of the structure.

\begin{tabular}{|c|c|c|c|c|c|c|c|}
\hline \multicolumn{2}{|c|}{ Seismic input } & \multicolumn{2}{|c|}{ EL Centro } & \multicolumn{2}{|c|}{ Taft } & \multicolumn{2}{|c|}{ Artifical wave } \\
\hline \multicolumn{2}{|c|}{ Acceleration } & Peak & RMS & Peak & RMS & Peak & RMS \\
\hline \multirow{5}{*}{$0.1 \mathrm{~g}$} & F model & 3.42 & 0.69 & 3.75 & 0.54 & 3.84 & 0.64 \\
\hline & FR model & 2.71 & 0.48 & 3.38 & 0.46 & 3.13 & 0.45 \\
\hline & FT model & 3.10 & 0.62 & 3.52 & 0.45 & 3.33 & 0.55 \\
\hline & $R_{F R}(\%)$ & 20.79 & 30.69 & 9.72 & 14.52 & 18.53 & 30.66 \\
\hline & $R_{F T}(\%)$ & 9.34 & 11.05 & 5.96 & 15.72 & 13.50 & 15.05 \\
\hline \multirow{5}{*}{$0.2 \mathrm{~g}$} & F model & 4.81 & 0.64 & 4.43 & 0.57 & 5.15 & 0.64 \\
\hline & FR model & 3.16 & 0.48 & 3.81 & 0.46 & 3.79 & 0.48 \\
\hline & FT model & 3.69 & 0.53 & 4.09 & 0.50 & 4.22 & 0.55 \\
\hline & $R_{F R}(\%)$ & 34.27 & 24.78 & 13.95 & 19.52 & 26.33 & 24.81 \\
\hline & $R_{F T}(\%)$ & 23.38 & 17.83 & 7.67 & 12.65 & 18.00 & 14.67 \\
\hline
\end{tabular}

The peak and root mean square (RMS) of the three models are compared and analyzed under three seismic wave excitations. Compared with the peak that reflects the response at a certain moment, the RMS can characterize the response over the whole period. Therefore, the RMS and peak of acceleration and relative displacement are used to characterize the damping effect of controlled and uncontrolled structures. The RMS is calculated by the following equation.

$$
R M S=\sqrt{\frac{1}{n} \sum_{i=1}^{n} x_{i}^{2}}
$$

where $x_{i}$ represents the response of the corresponding sampling point during the whole analysis; and $n$ is the total number of sampling points. The definition of the vibration reduction effect is as follows: vibration reduction effect $=$ (response of the uncontrolled structure - response of the controlled structure)/(response of the uncontrolled structure $) \times 100 \%$. That is

$$
R=\left(a_{F}-a_{F i}\right) / a_{F}
$$

where $a_{F}$ and $a_{F i}$ refer to the peak or root-mean-square values of the acceleration or relative displacement on the top floor of the uncontrolled structure and controlled structure, respectively; $R_{F R}$ and $R_{F T}$ represent the reduction effects of the FR model and FT model, respectively.

\subsubsection{Vibration-reduction effect of the acceleration}

The acceleration is an indicator of the structural energy attenuation and can be used to evaluate the damage degree of the structure. Therefore, the damping effects of the three models under different seismic wave excitations are compared and analyzed.

Table 3 lists the peak and RMS values of acceleration responses at the roof of the three models in different cases. The results show that the controlled structures can reduce the peak values and RMS of acceleration of the primary structure in all cases. For example, the FR model show a favorable and stable peak and RMS reduction effect $(9 \%$ $35 \%$ and $14 \%-31 \%$ ) under three seismic waves with two PGAs; Similarly, the peak and RMS reduction effect of FT model are $5 \%-24 \%$ and $11 \%-18 \%$ in the same cases, which suggests that the RW-TMDs have a better and more stable damping performance than that of the STMD. In addition, the reduction effects were favorable, especially under the EL Centro waves; For FR model, the $R_{F R}$ of peak and RMS of acceleration increased from $20.79 \%$ and $30.69 \%$ to $34.27 \%$ and $24.78 \%$ (marked in bold), respectively, with the increase of seismic wave intensities. Moreover, it is also observed that the damping effect of the controlled structure becomes increasingly obvious with increasing seismic intensity under other seismic excitations. These findings coincide with those found in previous studies ${ }^{42}$ in which the effectiveness of the TMDs is significant with an increase in PGAs. 
Table 4 Relative displacement responses at the roof of the structure.

\begin{tabular}{|c|c|c|c|c|c|c|c|}
\hline \multicolumn{2}{|c|}{ Seismic input } & \multicolumn{2}{|c|}{ EL Centro } & \multicolumn{2}{|c|}{ Taft } & \multicolumn{2}{|c|}{ Artifical wave } \\
\hline & & $\begin{array}{l}\text { Peak } \\
(\mathrm{mm})\end{array}$ & $\begin{array}{l}\text { RMS } \\
(\mathrm{mm})\end{array}$ & $\begin{array}{l}\text { Peak } \\
(\mathrm{mm})\end{array}$ & $\begin{array}{l}\text { RMS } \\
(\mathrm{mm})\end{array}$ & $\begin{array}{l}\text { Peak } \\
(\mathrm{mm})\end{array}$ & $\begin{array}{l}\text { RMS } \\
(\mathrm{mm})\end{array}$ \\
\hline \multirow{5}{*}{$0.1 \mathrm{~g}$} & F model & 9.02 & 3.25 & 9.70 & 3.76 & 11.53 & 4.10 \\
\hline & FR model & 6.18 & 2.48 & 8.22 & 2.60 & 7.53 & 3.10 \\
\hline & FT model & 7.12 & 2.20 & 8.55 & 3.00 & 8.31 & 2.73 \\
\hline & $R_{F R}(\%)$ & 31.48 & 23.87 & 15.18 & 30.76 & 34.69 & 24.41 \\
\hline & $R_{F T}(\%)$ & 21.09 & 32.36 & 11.84 & 20.20 & 27.89 & 33.35 \\
\hline \multirow{5}{*}{$0.2 \mathrm{~g}$} & F model & 22.22 & 8.23 & 22.90 & 8.08 & 24.01 & 9.68 \\
\hline & FR model & 10.69 & 2.98 & 17.02 & 5.64 & 14.35 & 6.43 \\
\hline & FT model & 15.02 & 5.12 & 18.15 & 5.94 & 17.04 & 5.56 \\
\hline & $R_{F R}(\%)$ & 51.91 & 63.83 & 25.67 & 30.25 & 40.26 & 33.61 \\
\hline & $R_{F T}(\%)$ & 32.42 & 37.75 & 20.74 & 26.57 & 29.05 & 42.60 \\
\hline
\end{tabular}

This is because the seismic damping mechanism is similar for the TMD system and the RW-TMDs proposed in this paper. That is, when the structure vibrates due to an earthquake excitation, a force will be generated to act on the TMDs system to drive the TMDs to move. Correspondingly, the TMDs with a large inertia effect will produce the reverse motion with respect to the structure, and then the inertia force will act on the main structure, thereby suppressing the vibration of the structure. Moreover, with increasing earthquake intensity, the inertial force becomes larger, and the vibration response of the main structure can be significantly suppressed.

\subsubsection{Vibration-reduction effect of the displacement}

Similar to the acceleration response, the $a_{F}$ and $a_{F i}$ of the relative displacement under different seismic waves are shown in Table 4. The results show that compared with the uncontrolled structure, the controlled structure transfers part of the structural input energy to the TMDs system for dispersion, greatly reducing the peak and RMS value of relative displacement. In particular, under EL Centro wave excitation with $\mathrm{PGA}=0.2 \mathrm{~g}$, the FR model and FT model have obvious reduction effect of peak value $(51.91 \%$ and $32.42 \%$ ) compared to the F model. Moreover, the reduction rate of the RMS value is also notable, reaching $63.83 \%$ and $37.75 \%$, respectively, demonstrating the effectiveness of RW-TMDs over the whole period.

In addition, the type of input seismic wave has a great influence on the damping performance of the TMD. For example, under Artificial wave excitation with $\mathrm{PGA}=0.1 \mathrm{~g}$, the FR and FT models exhibit obvious peak reduction rates of $34.69 \%$ and $27.89 \%$, respectively. However, as the seismic intensity increases, the peak reduction ratio under EL Centro excitation is more remarkable, i.e., from $31.48 \%$ to $51.91 \%$ for the FR model and from $21.09 \%$ to $32.42 \%$ for the FT model, which is consistent with the found in acceleration response. The reason is that the movement of the TMD system exists time-lag effects under seismic excitation. If the seismic excitation is small, the relative displacement between the TMD system and the main structure is insufficient, and then the force generated by the TMD system on the main structure is relatively small, thus the damping effect is not fully displayed. However, as the input seismic energy increases, the damping effect of TMD system is more significant.

It is interesting to note that the peak response of the FR model is significantly suppressed under Artificial wave excitation with different PGAs which is important for characterizing the effectiveness of the RW-TMDs. However, the $R_{F R}$ value of RMS $(24.41 \%$ and $33.61 \%)$ are slightly lower than that of the $R_{F T}$ value of RMS $(33.35 \%$ and $42.60 \%$ ) under two PGAs. This is mainly because the responses of the first mode were largest, while STMD was suspended on top of the structure, so the RMS value could be suppressed to a large degree over the entire time course. In addition, the vibration of dampers lagged behind the primary structure in general, while each RW-TMD was connected in parallel with the primary structure by six pairs springs and dampers, increasing the time lag of each TMD in the FR model. 
Therefore, compared with the reduction rate of acceleration response in Section 3.4.1, the control effect on the structural relative displacement response is better than the acceleration response. In addition, the parameter design of the TMD system is based on the minimum displacement, and the frequency of the TMD system is adjusted to the first modal frequency of the structure. Thus, the following analysis is mainly based on the displacement response.

\subsection{Comparative analysis of power in band}

The effectiveness of the RW-TMDs has been demonstrated in the time domain based on the peak and RMS values. In reference to the concept of power in band (PWB) used in the literature ${ }^{43}$, this paper further demonstrates the validity of RW-TMDs in the frequency domain. To facilitate understanding, its definition and formula are given as follows. The power in band (PWB) is a measure of the total power of the signal (response history) for a given frequency range $\left(f_{1}-f_{2}\right)$ defined by the following formula:

$$
P W B=\frac{\sum_{f_{1}}^{f_{2}}(M S)^{2}}{E N B W}
$$

where MS is the Fourier amplitude spectrum, and ENBW is the effective noise bandwidth.

To simplify the calculation, the rectangular window is considered and ENBW = 1. Therefore, Eq. (25) can be written as shown in Eq. (26).

$$
P W B=\int_{0}^{T}[f(t)]^{2} d t=\sum_{0}^{f_{n}}(M S)^{2}
$$

where $\mathrm{T}$ is the total duration of the time history, and $\mathrm{fn}$ is Nyquist's frequency.

When performing Fourier analysis on seismic waves, the components above the Nyquist frequency are undetectable, so the Nyquist frequency is generally chosen as the upper limit. Therefore, the equation is given as follow: $f=\frac{1}{2 \Delta t}$ (27), where $\Delta \mathrm{t}$ is the sampling interval of seismic waves.

The time interval of the seismic excitation in this article is $0.02 \mathrm{~s}$, and then the Nyquist frequency can be obtained as $25 \mathrm{~Hz}$. Therefore, to compare and analyze the effectiveness of the controlled structure, MATLAB software is used to calculate the PWB of the relative displacements on the top layer of the controlled structure and the uncontrolled structure under three different seismic excitations. The results are shown in Fig. 7. The PWB ratio of the relative displacement time history response of the FR model or FT model versus the $F$ model under different waves is represented by $\mathrm{P}$. Therefore, $\mathrm{P}_{\mathrm{FR}} 0.1 \mathrm{~g}$ and $\mathrm{P}_{\mathrm{FR}} 0.2 \mathrm{~g}$ represent the $\mathrm{PWB}$ ratio of the FR model to the $\mathrm{F}$ model under $\mathrm{PGA}=0.1 \mathrm{~g}$ and $0.2 \mathrm{~g}$, while the $\mathrm{P}_{\mathrm{FT}} 0.1 \mathrm{~g}$ and $\mathrm{P}_{\mathrm{FT}} 0.2 \mathrm{~g}$ represent the $\mathrm{PWB}$ ratio of the FT model to the $\mathrm{F}$ model under $\mathrm{PGA}=0.1 \mathrm{~g}$ and $0.2 \mathrm{~g}$. When $\mathrm{P}=1$, the added damping system has no effect on the response control of the structure. Conversely, the smaller the P-value, the better the effectiveness of the TMD system is.

Fig.7 shows that the controlled structure has a better damping effect than the uncontrolled structure. The P-value decreases with increasing input seismic intensity, further illustrating the effectiveness of the controlled structure. In particular, under EL Centro and Artificial wave excitations with $\mathrm{PGA}=0.2 \mathrm{~g}$, the FR model and FT model reach maximum reduction rates of $87.53 \%$ and $72.02 \%$. Moreover, the P-value of the FR model is larger than that of the FT model under Artificial wave excitation, which is consistent with the results of the reduction rate analysis of the RMS in the time domain.

Therefore, the comparative analysis in the time and frequency domains proves the effectiveness of the RWTMDs, while the RW-TMDs exhibit more stable damping effects compared to that of the STMDs overall.

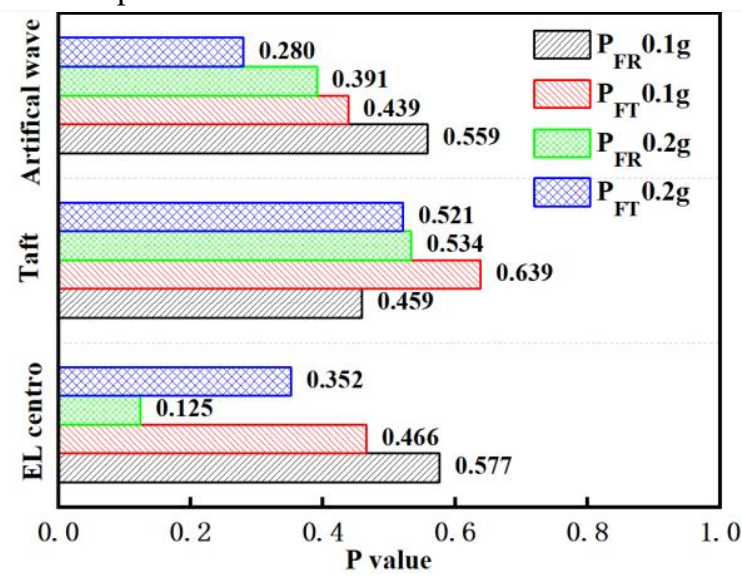

Fig. 7 Power in band ratio for relative displacement response.

\subsection{Comparative analysis of damping energy dissipation}

Putignano and Carbone ${ }^{44}$ reported that the assessment of energy dissipation and losses, in all their different forms, including, for example, friction, phase transitions, material hysteresis, is crucial in current applied science and engineering research. Besides, many studies have shown that the structures with TMDs can improve the capacity of damping energy dissipation, so the controlled structure consumes more damping energy than the uncontrolled structure ${ }^{45}$. According to the law of conservation of energy, energy does not disappear for no reason but can be transformed from one form to another. Therefore, when earthquake excitations with identical intensity and duration, the increase in the damping energy dissipation of the controlled structure will inevitably change the structural energy component; That is, the other energy components of the structure will be reduced, like the plastic energy. The reason is that compared to uncontrolled structures, the controlled structure with TMDs can store part of the input energy in the TMD, delay the energy release at the critical 


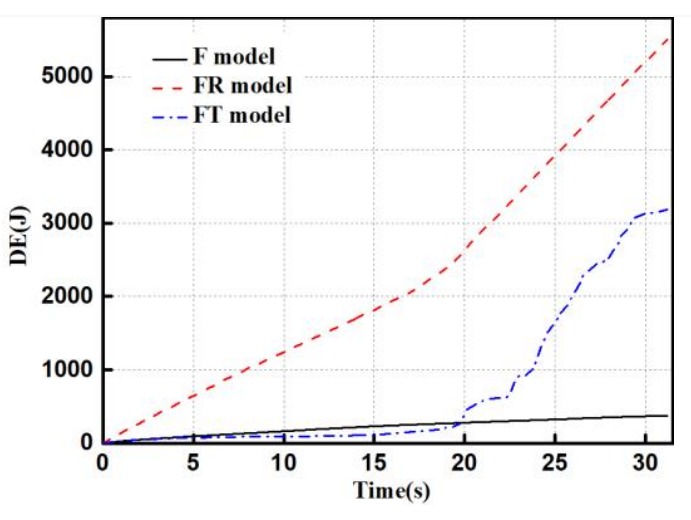

(a) Viscous damping energy

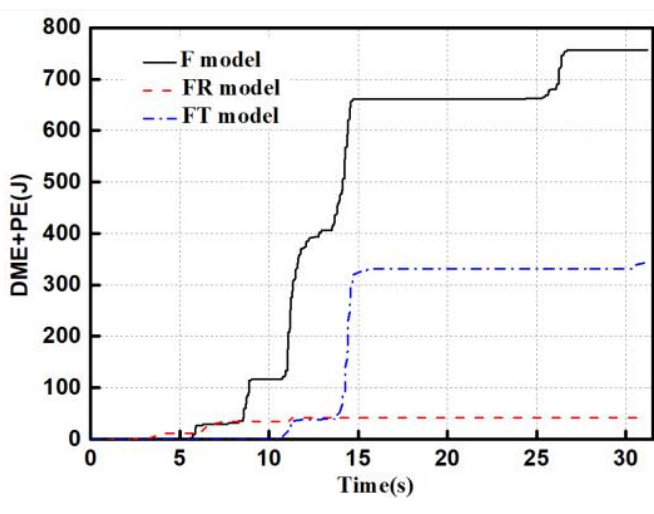

(b) Hysteretic energy

Fig. 8. Energy time histories of the six-story moment-resisting frame under white noise.

state, and subsequently release the stored energy in the form of damped energy dissipation, reducing the plastic energy ${ }^{46}$. While the magnitude of plastic energy dissipation is directly related to the damage degree of the structure, so the TMD system can effectively reduce the structural damage degree.

As reported by Lu et al. ${ }^{47}$, the damping effects of the TMD are mainly related to the frequency characteristics of excitation. Therefore, a comparative analysis of the controlled and uncontrolled structures under Gaussian white noise is conducted to reflect the effectiveness of the TMD from the perspective of energy. It is reported that input energy (IE) is converted into different energy forms in the structure, such as strain energy (SE), kinetic energy (KE), damping energy (DE), and plastic performance PE ${ }^{48}$. However, the SE and KE only participate in the conversion of energy, not in the dissipation of energy. Therefore, the plastic energy and damping energy are used to analyze the effectiveness of the TMD system. Fig. 8 (a-b) shows the time history curve of the viscous damping energy dissipation and the hysteretic energy dissipation for the structure with and without TMDs under the excitation of Gaussian white noise, where DE represents damping energy, DME represents damage energy, PE represents plastic energy, and the sum of DME and PE is the hysteresis energy, i.e., the inelastic strain energy.

Fig. 8(a) shows that the viscous damping energy of FR model and $\mathrm{F}$ model are the upper and lower limits. Correspondingly, Fig. 8(b) shows that the hysteresis energy of the uncontrolled structure is the largest compared to controlled structure. This is because a limited amount of damping energy is dissipated at a slow rate, so the input energy can only be dissipated through the nonlinear deformation of the material, i.e., plastic deformation, increasing plastic energy, and then leading to serious structural damage. In contrast, for controlled structures, the input seismic energy can be partially stored in the TMD system, and the stored energy is subsequently released slowly in the form of damped energy, reducing the plastic energy dissipation ${ }^{46}$.

Therefore, the TMDs is very effective in protecting structure from suffering major damage. Furthermore, Fig. 8 also shows that the RW-TMDs improved the frequency sensitivity compared to the STMDs due to the increased frequency tuning width of the two TMDs with different characteristics, which effectively exerts the tuning effect of multiple TMDs and increases the input energy stored in each TMD. Thus, the viscous damping energy of FR model is the largest and the hysteretic energy is the smallest compared with FT model and F model, indicating that the RW-TMDs have a better tuning effect. The above results and discussions are similar to the results of the study by Wong 46 .

\section{Conclusions}

This paper presents a multi-modal controlled tuned mass damper (TMD) in the form of a rocking wall, i.e., a rocking wall tuned mass dampers (RW-TMDs), formed by synthesizing the structural advantages of the rocking wall structure and the damping principle of the TMD system. The optimized design of RW-TMDs arranged symmetrically along the height of the structure can not only improve the frequency sensitivity of STMD but also solve the accuracy problem of MTMDs in determining the installation location and the number of TMDs. The feasibility of the proposed method has been verified via numerical simulations with and without RW-TMDs and STMD under white noise and three seismic excitations. The results show that the controlled structures can significantly attenuate the structural response, and they exhibit a good damping effect. The following conclusions are drawn from the results of the numerical study.

(1) The additional TMD system changes the vibration characteristics of the main structure, there are a shift in the natural frequency of the controlled structure. The modal analysis results show that the absolute values of the frequency shifts of first three modes for the RW-TMDs are $3.81 \%$, 26.15\%, $12.65 \%$; Similarly, the frequency shifts value for STMD are $15.37 \%, 4.86 \%, 4.26 \%$. The STMD system has better control for the first mode, while the RWTMDs system has the advantage of multi-modal control, especially for the second mode. 
(2) The frequency response curve of three structures demonstrated that the RW-TMDs have a comparable vibration control effect than the STMD under white noise excitation. Moreover, the maximum lateral displacements show that the FR model can not only attenuate the structural displacement response but also improve the inter-story deformation pattern, making the inter-story displacement tends to be uniform, preventing soft-story failure.

(3) Compared with the uncontrolled structure, the RWTMDs can effectively reduce structural peak and the RMS value. For FR model, the acceleration reduction rate of peak and RMS can reach 9\%-35\% and 14\%-31\%, respectively; The displacement reduction rate of peak and RMS can reach $15 \%-52 \%$ and $23 \%-64 \%$, respectively. Similarly, for FT model, the acceleration reduction rate of peak and RMS can reach $5 \%-24 \%$ and $11 \%-18 \%$, respectively; The displacement reduction rate of peak and RMS can reach $11 \%-33 \%$ and $20 \%-43 \%$, respectively, demonstrating the effectiveness of the RW-TMDs. Besides, the power in band also proves the validity of the RW-TMDs in the frequency domains.

(4) The RW-TMDs can significantly reduce the structural damage degree, compared to the STMD. The RW-TMDs with different dynamic characteristics can increase the frequency tuning width and improve the frequency sensitivity. Consequently, multiple TMDs can store input energy in each TMD, and then release the energy in the form of damping energy, reducing the plastic energy.

As an alternative to traditional TMDs, the effectiveness of the device proposed in this article has been proven through numerical simulations. The RW-TMDs not only has the effect of multi-modal control, but also is simple to install and easy to repair, and it can save valuable space on the top of the building. Therefore, the RW-TMDs possess a high potential for practical applications of new buildings or buildings in service.

\section{Acknowledgments}

This research was supported by the National Natural Science Foundation of China (51474045 and 51774173). The authors would like to sincerely thank for all the teachers, classmates and staves for technical guidance.

\section{Authors' contributions}

N.W led the development of the method with assistance from L.S.X., L.S.S, L.S.D., Y.H., B.C. All authors gave final approval for publication and agree to be held accountable for the work performed therein.

\section{Competing interests}

The authors declare no competing interests.

\section{Additional information}

Shasha Lu (E-mail: lilyherb@163.com) and Wei Nie (Email: nwstudy@163.com) are Co-Corresponding authors.

\section{References}

1 Lu, Z., Huang, B., Wang, Z. \& Zhou, Y. Experimental comparison of dynamic behavior of structures with a particle damper and a tuned mass damper. J Struct
Eng.144, 04018211;

10.1061/(asce)st.1943-541x.0002213 (2018).

2 Frahm, H. Device for damping vibration of bodies. (1909).

3 Yao, J. Concept of structural control. Asce Journal of the Structural Division. 98, 1567-1574;

10.1061/JSDEAG.0003280 (1972).

4 Elias, S. \& Matsagar, V. Research developments in vibration control of structures using passive tuned mass dampers. Annu Rev Control. 44, 129-156;

10.1016/j.arcontrol.2017.09.015 (2017).

5 Tang, X. \& Zuo, L. Simultaneous energy harvesting and vibration control of structures with tuned mass dampers. J Intel Mat Syst Str. 23, 2117-2127;

$10.1177 / 1045389 \times 12462644$ (2012).

6 Setareh, M., Ritchey, J. K., Baxter, A. J. \& Murray, T. M. Pendulum tuned mass dampers for floor vibration control. J Perform Constr Fac. 20, 64-73; 10.1061/(ASCE)08873828(2006)20:1(64) (2006).

7 Kerschen, G., Kowtko, J. J., Mcfarland, D. M., Bergman, L. A. \& Vakakis, A. F. Theoretical and experimental study of multimodal targeted energy transfer in a system of coupled oscillators. Nonlinear Dynam. 47, 285-309; 10.1007/s11071-006-9073-5 (2007).

8 Moon, K. S. Vertically distributed multiple tuned mass dampers in tall buildings: performance analysis and preliminary design. Struct Des Tall Spec. 19, 347-366; 10.1002/tal.499 (2010).

9 Igusa, T. \& Xu, K. Wide-band response of multiple subsystems with high modal density. (ed. Lin Y.K., Elishakoff I.)131-145 (Springer,Berlin, Heidelberg, 1991).

10 Yamaguchi, H. \& Harnpornchai, N. Fundamental characteristics of Multiple Tuned Mass Dampers for suppressing harmonically forced oscillations. Earthq Eng Struct D. 22, 51-62; 10.1002/eqe.4290220105 (1993).

11 Rana, R. \& Soong, T. T. Parametric study and simplified design of tuned mass dampers. Eng Struct. 20, 193-204; 10.1016/S0141-0296(97)00078-3 (1998).

$12 \mathrm{Li}, \mathrm{C}$. Performance of multiple tuned mass dampers for attenuating undesirable oscillations of structures under the ground acceleration. Earthq Eng Struct D. 29, 14051421;10.1002/1096-9845(200009)29:9<1405::Aideqe976>3.0.Co;2-4 (2000)

13 Guo, Y. Q. \& Chen, W. Q. Dynamic analysis of space structures with multiple tuned mass dampers. Eng Struct. 29, 3390-3403; 10.1016/j.engstruct.2007.09.004 (2007).

14 Petit, F., Loccufier, M. \& Aeyels, D. On the attachment location of dynamic vibration absorbers. J Vib Acoust. 131, 837-838; 10.1115/1.3085888 (2009).

15 Elias, S., Matsagar, V. \& Datta, T. K. Distributed tuned mass dampers for multi-mode control of benchmark building under seismic excitations. J Earthq Eng. 23, 1137-1172; 10.1080/13632469.2017.1351407 (2017).

16 Dehghan-Niri, E., Zahrai, S. M. \& Mohtat, A. Effectiveness-robustness objectives in MTMD system design: An evolutionary optimal design methodology. Struct Control Hlth. 17, 218-236; 10.1002/stc.297 (2010).

17 Eatherton, M. R. et al. Design concepts for controlled rocking of self-centering steel-braced frames. J Struct Eng. 140, 04014082;

10.1061/(ASCE)ST.1943-541X.0001047 (2014). 
18Zhou, Y. \& Lu, X. State-of-the-art on rocking and selfcentering structures. Journal of Building Structures. 32, 1-10; 10.1111/j.1759-6831.2010.00113.x (2011).

19Roh, H. \& Reinhorn, A. M. Modeling and seismic response of structures with concrete rocking columns and viscous dampers. Eng Struct. 32, 2096-2107; 10.1016/j.engstruct.2010.03.013 (2010).

20 Vassiliou, M. F. \& Makris, N. Dynamics of the vertically restrained rocking column. J Eng Mech. 141, 04015049; 10.1061/(asce)em.1943-7889.0000953 (2015).

21 Wada, A., Qu, Z., Motoyui, S. \& Sakata, H. Seismic retrofit of existing SRC frames using rocking walls and steel dampers. Frontiers of Architecture and Civil Engineering in China. 5, 259-266; 10.1007/s11709-0110114-x (2011).

22 Qu, Z., Wada, A., Motoyui, S., Sakata, H. \& Kishiki, S. Pin-supported walls for enhancing the seismic performance of building structures. Earthq Eng Struct D. 41, 2075-2091; 10.1002/eqe.2175 (2012).

23 Joshi, A. S. \& Jangid, R. S. Optimum parameters of multiple tuned mass dampers for base-excited damped systems. J Sound Vib. 202, 657-667;

10.1006/jsvi.1996.0859 (1997).

24 Ok, S.Y., Song, J. \& Park, K.-S. Development of optimal design formula for bi-tuned mass dampers using multiobjective optimization. J Sound Vib. 322, 60-77; 10.1016/j.jsv.2008.11.023 (2009).

25 Yang, H. Performance analysis of semi-rigid connections in prefabricated high-rise steel structures. Structures. 28, 837-846; 10.1016/j.istruc.2020.09.036 (2020).

26 Domizio, M., Ambrosini, D. \& Curadelli, O. Performance of tuned mass damper against structural collapse due to near fault earthquakes. J Sound Vib. 336, 32-45; 10.1016/j.jsv.2014.10.007 (2015).

27 Jangid, R. S. Optimum multiple tuned mass dampers for base-excited undamped system. Earthq Eng Struct D. 28, 1041-1049; 10.1002/(sici)1096-9845(199909)28:9<1041::Aideqe853>3.0.Co;2-e (1999).

28 Hwang, J., Lee, S. \& Kareem, A. Estimation of modal masses of a structure with a mass-type damping device. Eng Struct. 99, 149-156; 10.1016/j.engstruct.2015.04.054 (2015).

29 Tharwat \& Sakr, A. Vibration control of buildings by using partial floor loads as multiple tuned mass dampers. Hbrc Journal. 30, 188-198; 10.1016/j.hbrcj.2015.04.004 (2015).

30 Matta, E. Effectiveness of tuned mass dampers against ground motion pulses. J Struct Eng. 139, 188-198; 10.1061/(ASCE)ST.1943-541X.0000629 (2013).

31 Macrae, G. A., Kimura, Y. \& Roeder, C. Effect of Column Stiffness on Braced Frame Seismic Behavior. J Struct Eng. 130, 381-391; 10.1061/(ASCE)07339445(2004)130:3(381) (2004).

$32 \mathrm{Qu}, \mathrm{Z}$. Study on seismic damage mechanism control and design of rocking wall-frame structures. Dissertation, Tsinghua University, (2010).

33 Yan, S. Y. L., Jia, J. \& Yu, D. Influence of rocking wall stiffness on seismic behavior of frame rocking wall structure. World earthquake engineeing. 30, 27-33;
(2014).

34 Chen, Y. H. \& Huang, Y. H. Timoshenko beam with tuned mass dampers and its design curves. J Sound Vib. 278, 873-888; 10.1016/j.jsv.2003.10.013 (2004).

$35 \mathrm{Nie}, \mathrm{W}$. et al. Seismic performance analysis of rocking wall TMDs structure based on shaking table test. Earthq Struct. 21, 029-042; 10.12989/eas.2021.21.1.029 (2021).

36 Au-Yeung, K. Y., Yang, B., Sun, L., Bai, K. \& Yang, Z. Super Damping of Mechanical Vibrations. Sci Rep-UK. 9, 10.1038/s41598-019-54343-3 (2019).

$37 \mathrm{Li}$, K. N. \& Darby, A. P. Experiments on the effect of an impact damper on a multiple-degree-of-freedom system. J Vib Control. 12, 445-464; 10.1177/1077546306063504 (2006).

$38 \mathrm{Lu}$, Z., Chen, X., Zhang, D. \& Dai, K. Experimental and analytical study on the performance of particle tuned mass dampers under seismic excitation. Earthq Eng Struct D. 46, 697-714; 10.1002/eqe.2826 (2016).

39 MoT (2008), Guideline for seismic design of highway bridges,JTG/TB02-01-2008.Beijing,Ministryof Transport of the People's Republic of China; Beijing, China.

40Zhou, Y.L., Han, Q., Du, X.L. \& Jia, Z.l. Shaking table tests of post-tensioned rocking bridge with doublecolumn bents. J Bridge Eng. 24, 04019080; 10.1061/(asce)be.1943-5592.0001456 (2019).

41 Kechidi, S., Colaço, A., Alves Costa, P., Castro, J. M. \& Marques, M. Modelling of soil-structure interaction in OpenSees: A practical approach for performance-based seismic design. Structures. 30, 75-88; 10.1016/j.istruc.2021.01.006 (2021).

$42 \mathrm{Lu}$, Z., Chen, X., Zhang, D. \& Dai, K. Experimental and analytical study on the performance of particle tuned mass dampers under seismic excitation. Earthq Eng Struct D. 46, 697-714; 10.1002/eqe.2826 (2017).

43 Rakicevic, Z. T., Bogdanovic, A., Jurukovski, D. \& Nawrotzki, P. Effectiveness of tune mass damper in the reduction of the seismic response of the structure. B Earthq Eng. 10, 1049-1073; 10.1007/s10518-012-9341-3 (2012).

44 Putignano, C. \& Carbone, G. Viscoelastic Damping in alternate reciprocating contacts. Sci Rep-UK. 7, 8333; 0.1038/s41598-017-08507-8 (2017).

45 Elias, S., Matsagar, V. \& Datta, T. K. Effectiveness of distributed tuned mass dampers for multi-mode control of chimney under earthquakes. Eng Struct. 124, 1-16; 10.1016/j.engstruct.2016.06.006 (2016).

46 Wong, K. K. Seismic Energy Dissipation of Inelastic Structures with Tuned Mass Dampers. J Eng Mech. 134, 163-172;10.1061/(asce)0733-9399(2008)134:2(163) (2008).

47 Lu, Z., Huang, B., Wang, Z. \& Zhou, Y. Experimental Comparison of Dynamic Behavior of Structures with a Particle Damper and a Tuned Mass Damper. J Struct Eng. 144, 10.1061/(ASCE)ST.1943-541X.0002213 (2018).

48 Wong, K. K. F. \& Harris, J. L. Seismic damage and fragility analysis of structures with tuned mass dampers based on plastic energy. Struct Des Tall Spec. 21, 296310; 10.1002/tal.604 (2012). 